\title{
Enriquecimiento en Si mediante PVD de chapas magnéticas convencionales para aplicaciones a altas frecuencias
}

\author{
J. MOLINA ALDAREGUÍA ${ }^{1}$, C. GARCÍA-ROSALES ${ }^{2}$ Y J. GIL SEVILLANO ${ }^{2}$ \\ ${ }^{1}$ Department of Materials Science and Metallurgy, University of Cambridge, Cambridge CB2 3QZ, U.K. \\ ${ }^{2}$ Centro de Estudios e Investigaciones Técnicas de Guipúzcoa (CEIT) y Escuela Superior de Ingenieros Industriales (Universidad de Navarra) \\ Paseo de Manuel Lardizábal, 15, 20018 San Sebastián
}

\begin{abstract}
El presente trabajo estudia la viabilidad de un método para enriquecer en Si chapas de Fe-3\%Si convencionales, depositando Si en su superficie mediante PVD y sometiéndolas a un tratamiento térmico de difusión. Se trata de obtener chapas magnéticas con un contenido en Si de un 6,5\% en peso, ya que éstas presentan mayor resistividad y magnetoestricción nula, reduciendo las pérdidas y solucionando los problemas de ruido a altas frecuencias.

Las capas depositadas por PVD, de espesores $\sim 5 \mu \mathrm{m}$, presentan buena adherencia al substrato. En cuanto al tratamiento térmico, se observa que hay una velocidad de calentamiento crítica entre $5 \mathrm{y} 15^{\circ} / \mathrm{min}$, por encima de la cual la película se despega sin llegarse a formar intermetálicos en la intercara. A $3^{\circ} / \mathrm{min}$ se dan las condiciones para la formación de intermetálicos. Calentando directamente hasta temperaturas de unos $800^{\circ} \mathrm{C}$ se obtiene una capa superficial de $\mathrm{Fe}_{3} \mathrm{Si}\left(\alpha_{1}\right)$ que, aun siendo frágil, no se despega durante el tratamiento. Un calentamiento posterior hasta temperaturas del orden de $1200^{\circ} \mathrm{C}$ permite la homogeneización de la composición de la chapa. Existe evidencia de efecto Kirkendall en el par de difusión $\left.\mathrm{Fe}_{3} \mathrm{Si} \alpha_{1}\right) / \mathrm{Fe}(\alpha)$, debido a que la difusión de $\mathrm{Fe}$ en $\mathrm{Fe}_{3} \mathrm{Si}\left(\alpha_{1}\right)$ es mayor que la de $\mathrm{Si}$ en $\mathrm{Fe}(\alpha)$.
\end{abstract}

Palabras clave: Material magnético blando, pérdidas magnéticas, PVD, difusión.

Si enrichment of conventional electrical steel by PVD for high frequency applications.

The present work deals with the viability of a method for Si enrichment of conventional Fe-3\%Si sheets, by means of physical vapour deposition of $\mathrm{Si}$ at its surface and subsequent diffusion heating. The aim is to obtain magnetic sheets with a Si content of $6.5 \mathrm{wt} \%$, for they show higher resistivity and null magnetostriction, leading to lower energy losses and solving the noise problems at high frequencies.

The PVD deposited films, with $\sim 5 \mu \mathrm{m}$ thickness, show good adhesion to the substrate. Concerning the heat treatment, there is a critical heating rate between 5 and $15^{\circ} / \mathrm{min}$, above which delamination of the film is observed without formation of intermetallics at the interface. At $3 \% \mathrm{~min}$ the conditions for the formation of intermetallics are given. Direct heating up to $800^{\circ} \mathrm{C}$ leads to the formation of a $\mathrm{Fe}_{3} \mathrm{Si}\left(\alpha_{1}\right)$ surface layer, which, being brittle, does not delaminate during the heating process. Subsequent heating up to temperatures around $1200^{\circ} \mathrm{C}$ allows the homogenisation of the sheet composition. There is evidence of Kirkendall effect in the diffusion couple $\mathrm{Fe}_{3} \mathrm{Si}\left(\alpha_{1}\right) / \mathrm{Fe}(\alpha)$, due to the fact that the diffusion of $\mathrm{Fe}$ in $\mathrm{Fe}{ }_{3} \mathrm{Si}\left(\alpha_{1}\right)$ is faster than the diffusion of $\mathrm{Si}$ in $\mathrm{Fe}(\alpha)$.

Keywords: Soft magnetic material, magnetic energy losses, PVD, diffusion.

\section{INTRODUCCIÓN}

Existen varios motivos importantes que llevan a considerar el desarrollo de materiales magnéticos de bajas pérdidas: por una parte, se estima que el 5\% de la energía eléctrica generada es disipada por pérdidas dentro de los núcleos de los transformadores. Por otro lado, aunque se observa un progreso creciente en la tecnología semiconductora, tal avance se ve frenado por la imposibilidad de miniaturizar los núcleos de transformadores elaborados con chapa magnética convencional de $\mathrm{Fe}-3 \% \mathrm{Si}$, ya que a frecuencias elevadas las pérdidas magnéticas y los problemas de ruido son considerables $(1,2)$. En este contexto, la aleación Fe-6,5\% Si presenta la ventaja de poseer una mayor resistividad y permeabilidad que el Fe-3\% Si convencional, y por lo tanto menores pérdidas por corrientes Eddy y por histéresis, especialmente a altas frecuencias. Por otro lado, la mag- netoestricción de este material es prácticamente nula, lo que permite solucionar los problemas de ruido (3).

Sin embargo, es bien conocido que para contenidos en $\mathrm{Si}$ superiores al $4 \%$ en peso, las aleaciones Fe-Si tienen carácter frágil, no pudiendo ser procesadas por técnicas convencionales de laminación. En los últimos años se ha desarrollado con éxito un método de fabricación de chapas finas de Fe-6,5\% mediante deposición química en fase vapor (CVD), ya comercializado $(4,5)$. El presente trabajo estudia la viabilidad de un método de elaboración de chapas de $\mathrm{Fe}-6,5 \% \mathrm{Si}$, partiendo de chapas convencionales de Fe-3\% Si sobre las que se deposita Si mediante deposición física en fase vapor (PVD) y a las que se somete seguidamente a un tratamiento térmico de difusión. 


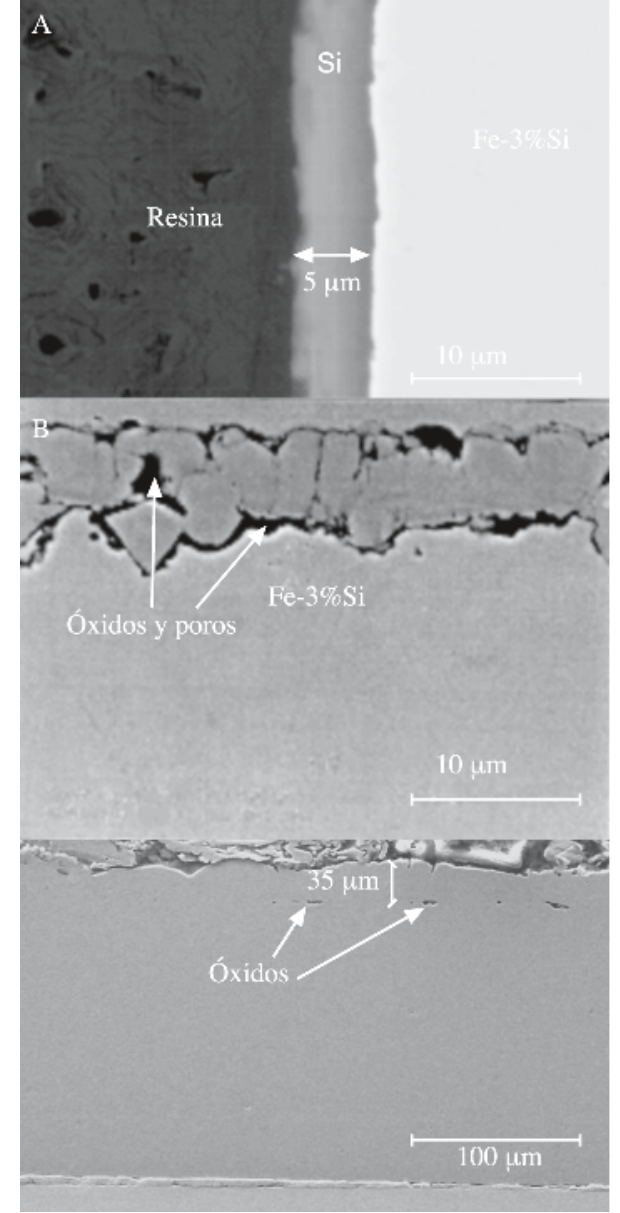

Micrografías de SEM de secciones transversales: (A) Capa de $\mathrm{Si}$ tras la deposición, (B) chapa tras tratamiento térmico a $800^{\circ} \mathrm{C}$ durante 30 min., (C) chapa tras ciclo térmico de la fig. 3 hasta una temperatura máxima de $1230^{\circ} \mathrm{C}$ durante $10 \mathrm{~min}$.

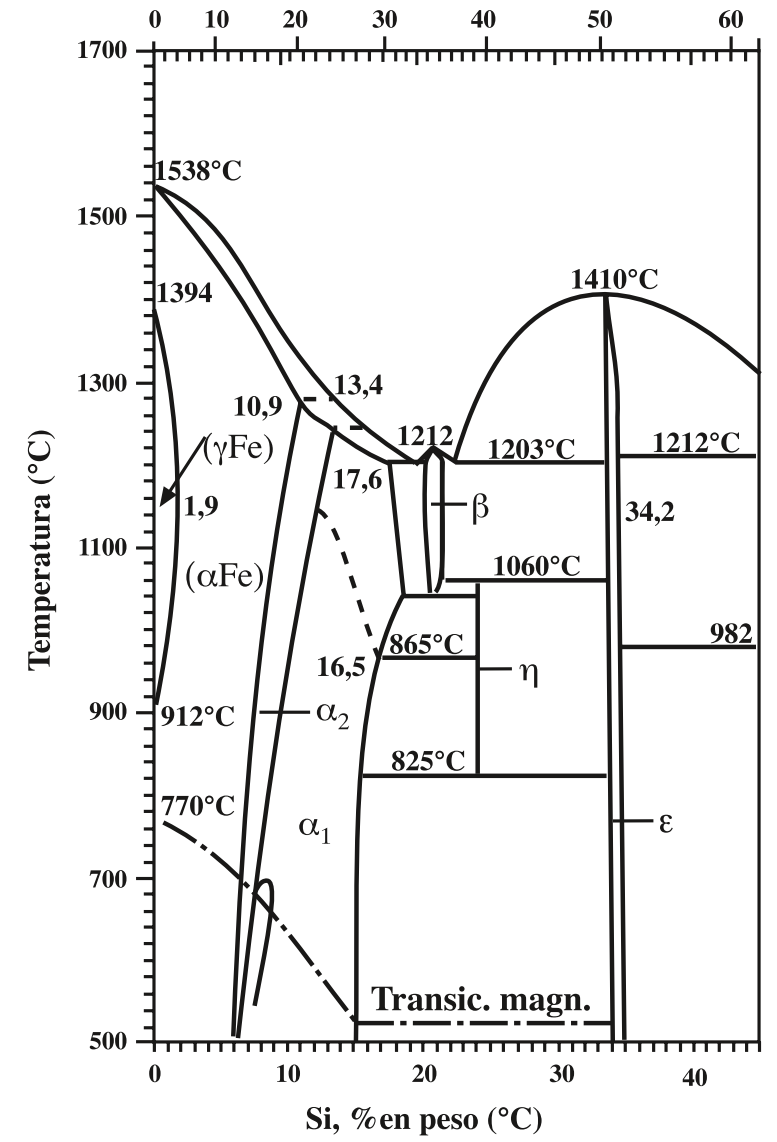

Figura 2: Zona rica en Fe del diagrama de fases Fe-Si (6)

\section{PROCEDIMIENTO EXPERIMENTAL}

Se han depositado películas de Si (grosor $1.4-4.2 \mu \mathrm{m}$ ) sobre chapas de Fe-3\%Si de 10 x $100 \mathrm{~mm}^{2}$ y 0,15-0,3 mm de grosor mediante DC magnetron sputtering a presiones del orden de 0,2 Pa y temperaturas del substrato entre $150 \mathrm{y}$ $200^{\circ} \mathrm{C}$. Se han empleado dos tipos de substrato: acero magnético de grano no orientado (N.O.) con contenido en Si del $3 \%$ en peso, y acero magnético de grano orientado (G.O) con un $2.8 \%$ en peso de Si. Previamente a la deposición, las chapas fueron pulidas mecánicamente con el fin de eliminar la capa de óxido superficial, e introducidas en baños de ultrasonidos de acetona, tricloroetileno y etanol, para eliminar impurezas y grasa. Durante los primeros minutos de deposición se utilizó una fuente $R F$ para asegurar una buena adherencia de la capa al substrato. Tras la deposición, las chapas fueron sometidas a tratamiento térmico a temperaturas entre 800 y $1230^{\circ} \mathrm{C}$ en atmósfera de Ar, con velocidades de calentamiento entre 2,5 y $4^{\circ} / \mathrm{min}$. Los tratamientos se han realizado en dos tipos de hornos: un horno de radiación y un horno de resistencias. En ambos casos, la muestra se introduce en un tubo de cuarzo, con circulación de Ar entre los extremos. El control de temperatura se realizó mediante un termopar tipo $R$ soldado a la muestra. La composición y microestructura de las chapas tras la deposición y tratamiento térmico se analizaron mediante un microscopio electrónico de barrido, MEB, equipado con EDAX.

\section{RESULTADOS Y DISCUSIÓN}

\subsection{Capas de Silicio Depositadas}

En la figura 1A se muestra una micrografía de MEB de la sección transversal de una capa de Si depositada de $5 \mu \mathrm{m}$ de espesor. La densidad de la capa es del orden del 80-90\% de la densidad teórica. La adherencia capa-substrato es muy buena, no observándose en la intercara porosidad ni restos de óxidos que pudiesen inhibir el subsiguiente proceso de difusión.

\subsection{Tratamiento Térmico de Difusión}

A velocidades de calentamiento superiores a $15^{\circ} / \mathrm{min}$ se produce el despegue de la capa de Si antes de que se produzca la formación de ningún compuesto intermetálico, debido a la diferencia en los coeficientes de dilatación térmica del substrato y del Si. Con rampas de calentamiento entre 2,5 y $4^{\circ} /$ min no se produce despegue, por lo menos hasta $800^{\circ} \mathrm{C}$, dándose las condiciones para que se puedan formar intermetálicos de Fe-Si en la intercara durante el calentamiento.

En la figura $1 \mathrm{~B}$ se muestra la sección transversal de una chapa calentada a $2,5^{\circ} / \mathrm{min}$ hasta $800^{\circ} \mathrm{C}$ en $\mathrm{Ar}$ y mantenida a esa temperatura durante 30 minutos. La capa de Si inicial, de $4,5 \mathrm{~mm}$ de espesor, ha reaccionado con el substrato, dando lugar a una capa superficial de $\mathrm{Fe}_{3} \mathrm{Si}\left(\alpha_{1}\right)$ (ver diagrama de fases Fe-Si, figura 2 ) de $\sim 8 \mu \mathrm{m}$ de espesor. Como puede observarse, 


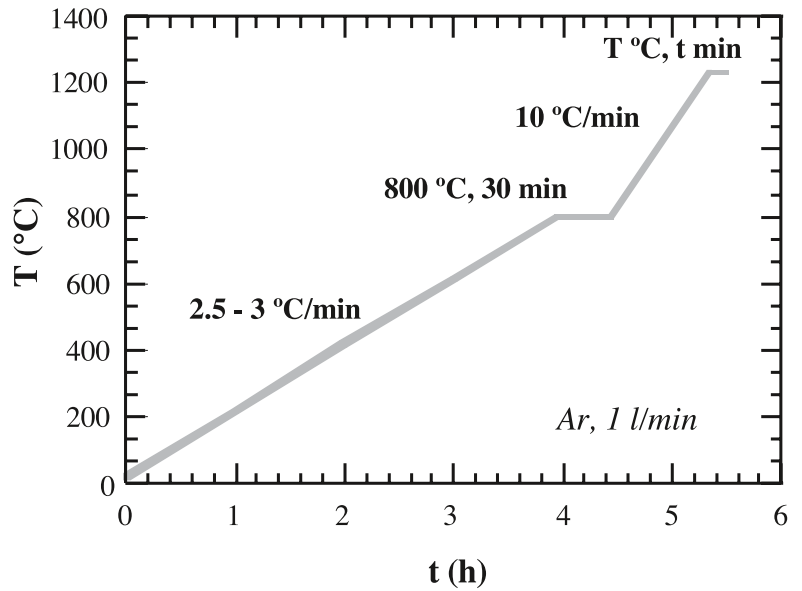

Figura 3: ciclo de calentamiento con el que se han obtenido los mejores resultados en cuanto a adherencia y homogeneidad.

esta capa está parcialmente despegada del substrato, lo cual se explica por tratarse de una fase muy frágil, pudiendo producirse el despegue al pulir mecánicamente las muestras. La composición de la capa, determinada mediante EDAX, es de 25at.\% Si y constante en toda la capa. No se ha detectado enriquecimiento en Si del substrato. Según se deduce del balance de masas, no ha habido pérdida de Si durante el tratamiento. Estos resultados concuerdan con los aportados por Zhang et al. (7), según los cuales el $\mathrm{Fe}_{3} \mathrm{Si}$ estequiométrico es más estable termodinámicamente que el $\mathrm{Fe}_{3} \mathrm{Si}$ no estequiométrico (rango de composición 6,5 - 14,35\% Si en peso).

A igual velocidad de calentamiento y temperaturas finales entre 800 y $1100^{\circ} \mathrm{C}$ se produce el despegue de la capa durante el calentamiento, debido a la formación de intermetálicos frágiles ricos en $\mathrm{Si}$ como $\mathrm{FeSi}(\varepsilon)$ y $\mathrm{Fe}_{2} \mathrm{Si}\left(\zeta_{\mathrm{b}}\right)$ (ver diagrama de fases, figura 2). Por tanto, es preferible realizar un mantenimiento por debajo de $825^{\circ} \mathrm{C}$, para dar tiempo a que se forme la fase $\mathrm{Fe}_{3} \mathrm{Si}\left(\alpha_{1}\right)$ y evitar el despegue durante el calentamiento.

Para producir el enriquecimiento en Si deseado, una vez formada la fase $\mathrm{Fe}_{3} \mathrm{Si}\left(\alpha_{1}\right)$, es preciso elevar la temperatura hasta $1100-1250^{\circ} \mathrm{C}$. Los ensayos realizados calentando hasta $1100^{\circ} \mathrm{C}$ muestran que la anterior capa de $\mathrm{Fe}_{3} \mathrm{Si}\left(\alpha_{1}\right)$ formada a $800^{\circ} \mathrm{C}$ es ahora más pobre en $\mathrm{Si}$, correspondiendo a la fase $\mathrm{Fe}_{3} \mathrm{Si}$ no este- quiométrica. Esto demuestra que a $1100^{\circ} \mathrm{C}$ la interdifusión ha comenzado. En la figura 3 se muestra el ciclo térmico con el que se han obtenido los mejores resultado en cuanto a adherencia y homogeneización.

En la figura 1C se observa la sección transversal de una chapa N.O. calentada según el ciclo de la fig. 3 hasta una temperatura final de $1230^{\circ} \mathrm{C}$ durante $10 \mathrm{~min}$. Los perfiles de concentración correspondientes a chapas N.O. y G.O. calentadas a esta temperatura se muestran en las figuras $4 a$ y $4 b$, en las que se observa una composición homogénea entre 3,5 y 4\% Si, en concordancia con el balance de masa. La capa inicial de Si y el grosor de la chapa eran de $4,2 \mu \mathrm{m}$ y $0,15 \mathrm{~mm}$ respectivamente para la chapa N.O., y de $5 \mu \mathrm{m}$ (en ambas caras) y $0,30 \mathrm{~mm}$ respectivamente para la chapa G.O. En ambos casos, el balance de masas hace prever una composición de $3,6 \%$ en peso de $\mathrm{Si}$, una vez homogeneizada la chapa. Por otra parte, en la imagen de la fig. 1C se observan a unas $35 \mu \mathrm{m}$ de la superficie una fina capa de óxidos y poros. Se cree que estos óxidos marcan la posición inicial de la intercara Si-substrato. Como el grosor de la capa de Si era de $4,2 \mu \mathrm{m}$, la existencia de estos óxidos a una profundidad de $35 \mu \mathrm{m}$ sólo puede deberse al efecto Kirkendall.

Si la difusión de $\mathrm{Fe}$ en $\mathrm{Fe}_{3} \mathrm{Si}$ es mucho más rápida que la difusión de Si en la solución sólida desordenada $\alpha$, se producirá un mayor flujo de átomos de Fe desde el substrato hacia la capa que de átomos de Si desde la capa hacia el substrato, dando lugar al desplazamiento de la intecara inicial hacia el substrato. Los coeficientes de difusión encontrados en la bibliografía $(8,9)$, que se muestran en la tabla I y se representan en la figura 5 para mayor claridad, ratifican estos resultados.

A $800^{\circ} \mathrm{C}$ la difusión de $\mathrm{Fe}$ en la fase ordenada $\mathrm{Fe}_{3} \mathrm{Si}\left(\alpha_{1}\right)$ es cuatro órdenes de magnitud mayor que la difusión del Si en la misma fase y en la fase desordenada $\mathrm{Fe}(\alpha)$. Al ir disminuyendo el contenido en $\mathrm{Si}$ del $\mathrm{Fe}_{3} \mathrm{Si}\left(\alpha_{1}\right)$, entrando en la región no estequiométrica, los coeficientes de difusión de Fe y Si en esta fase se van acercando a los valores que tienen en la fase desordenada Fe-Si. Lo mismo ocurre a $1125^{\circ} \mathrm{C}$. La razón por la que la difusión del componente mayoritario del $\mathrm{Fe}_{3} \mathrm{Si}$ es mucho más rápida que la del componente minoritario es su ordenamiento dentro de la estructura del $\mathrm{Fe}_{3} \mathrm{Si}(10,11)$, ya que los átomos de Si están rodeados de ocho átomos de Fe y no pueden saltar a posiciones vecinas sin alterar el orden de la estructura, mientras que los átomos de Fe sí pueden.
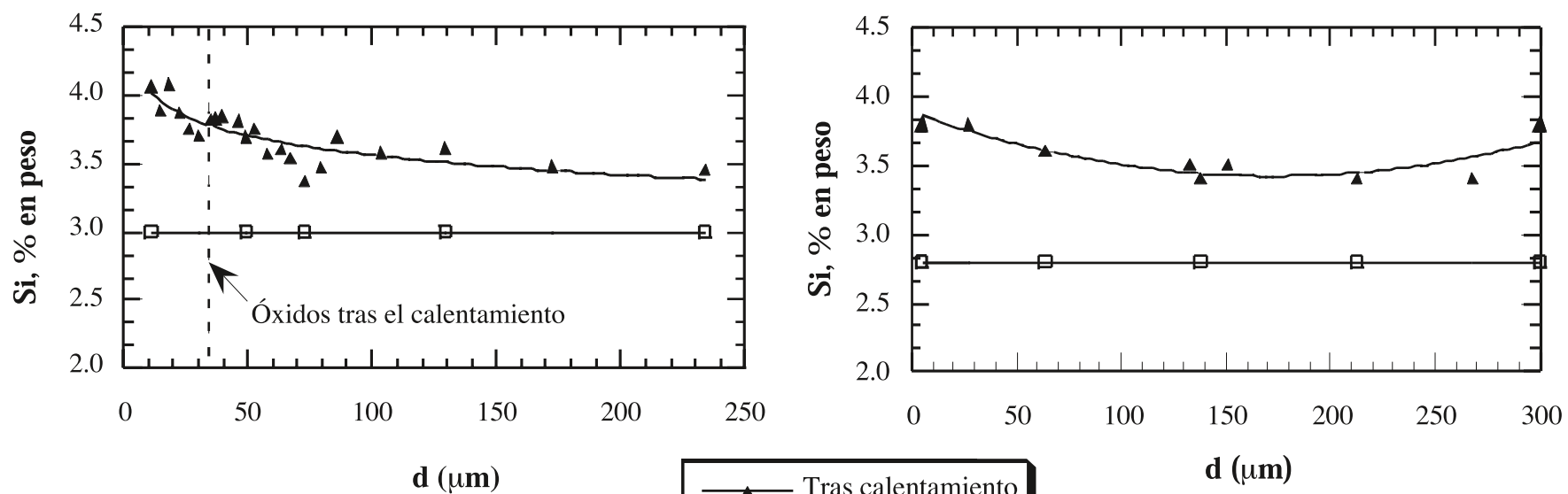

d $(\mu \mathbf{m})$

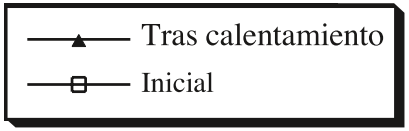

d ( $\mu \mathrm{m})$

Figura 4: Perfil de composición de a) chapa N.O. depositada por una cara, y b) chapa G.O. depositada por las dos cara, después del ciclo térmico de la fig. 3 hasta $1230^{\circ} \mathrm{C}$ durante 10 y 60 minutos respectivamente. 

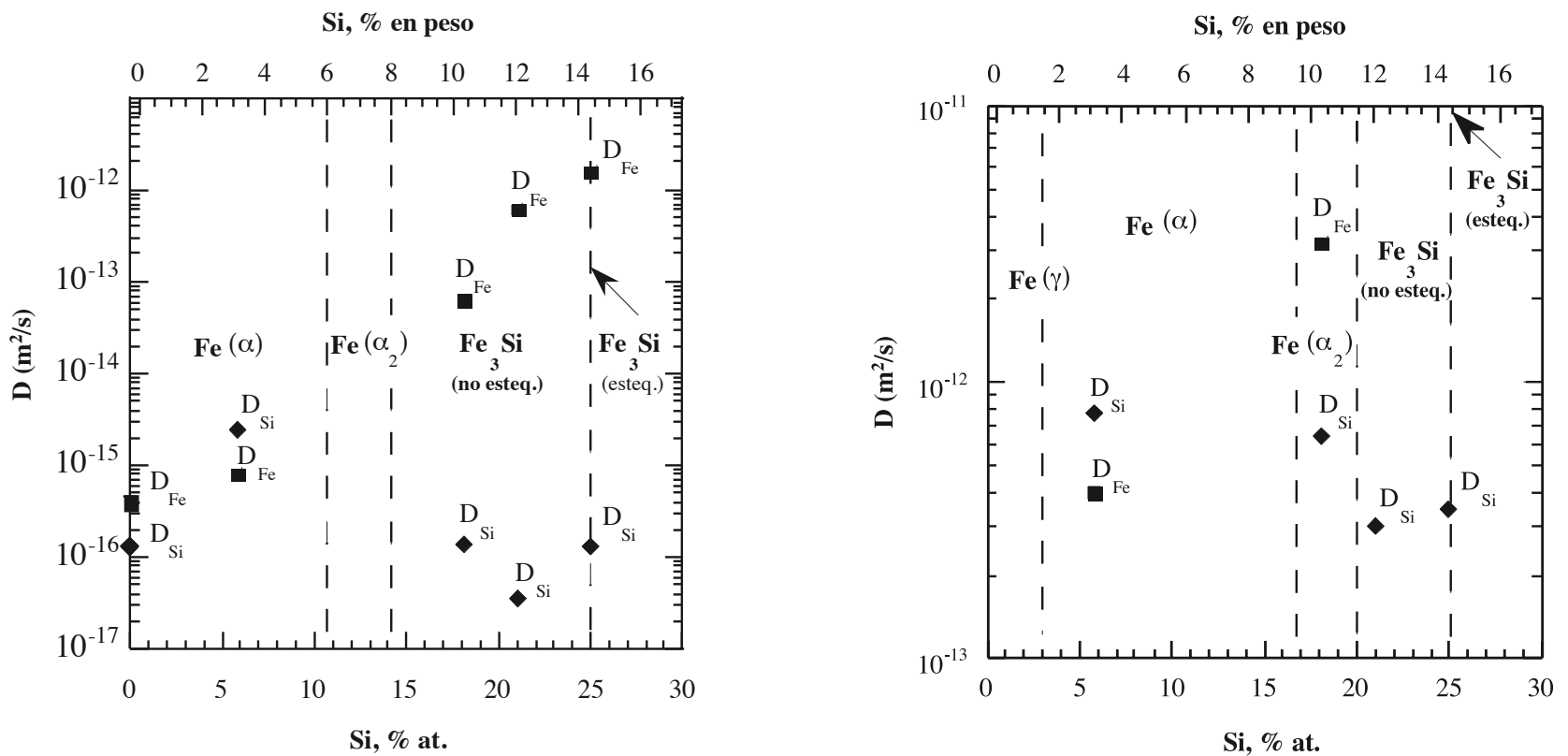

Figura 5: Coeficientes de difusión intrínsecos de $\mathrm{Si}(\mathrm{u})$ y Fe (n) en las fases ordenadas [8] y desordenadas [9] de las aleaciones Fe-Si a (a) $800^{\circ} \mathrm{C} \mathrm{y}$ (b) $1125^{\circ} \mathrm{C}$. Las líneas discontinuas representan los límites entre las regiones de estabilidad de cada fase, según el diagrama de fases de la figura 2.

\section{CONCLUSIONES}

La deposición física desde fase vapor mediante un sistema de DC-magnetron sputttering permite depositar capas de Si sobre chapas de $\mathrm{Fe}-3 \% \mathrm{Si}$ de varios mm de grosor, densidad de 80-90\% de la densidad teórica y buena adhesión al substrato.

Existe una velocidad de calentamiento crítica entre 5 y $15^{\circ} /$ min, por encima de la cual se produce el despegue de la capa de $\mathrm{Si}$, antes de que se pueda formar ningún compuesto intermetálico.

Una velocidad de calentamiento de $3 \%$ min permite la formación de compuestos intermetálicos en la intercara capasubstrato. Los mejores resultados se obtienen cuando se forma una capa uniforme de $\mathrm{Fe}_{3} \mathrm{Si}$ en la superficie a temperaturas por debajo de $800^{\circ} \mathrm{C}$. Aunque la fase ordenada $\mathrm{Fe}_{3} \mathrm{Si}\left(\alpha_{1}\right)$ presenta una fragilidad inherente que dificulta la reproducibilidad del proceso, cualquier intento de calentar la muestra por encima de $800^{\circ} \mathrm{C}$ sin haber dado tiempo a que se forme $\mathrm{Fe}_{3} \mathrm{Si}$ da lugar al despegue de la capa durante el calentamiento.

Una vez formada la capa superficial de $\mathrm{Fe}_{3} \mathrm{Si}$, un calentamiento hasta temperaturas entre 1150 y $1230^{\circ} \mathrm{C}$ permite la homogeneización de la composición de la chapa.

En el par de difusión $\mathrm{Fe}_{3} \mathrm{Si}\left(\alpha_{1}\right) / \mathrm{Fe}(\alpha)$ a temperaturas entre 1150 y $1230^{\circ} \mathrm{C}$ se produce el efecto Kirkendall, prueba de que el coeficiente de difusión del $\mathrm{Fe}$ en $\mathrm{Fe}_{3} \mathrm{Si}$ es mayor que el coeficiente de difusión del $\mathrm{Si}$ en la solución sólida desordenada $\alpha$.

\section{AGRADECIMIENTOS}

Los autores agradecen al Gobierno Vasco su apoyo para este proyecto en el marco de la Comunidad de Trabajo de los Pirineos (proyecto IT96/4). Jon Molina Aldareguia agradece al Departamento de Educación del Gobierno Vasco la concesión de una beca para participar en este proyecto. También queremos expresar nuestro agradecimiento a la empresa Siderúrgica Aristráin S.A. por proporcionarnos chapa de acero magnético N.O. Fe-3\%Si, y a la empresa OASA Transformadores XXI S.L. por proporcionarnos chapa magnética G.O. Fe-3\%Si así como
TABLA I: COEFICIENTES DE DIFUSIÓN DE Fe Y Si A 800 Y $1100^{\circ} \mathrm{C}$ EN LAS FASES Fe-Si ORDENADAS (8) Y DESORDENADAS (9)

\begin{tabular}{|l|l|l|l|l|l|l|}
\hline \multirow{2}{*}{ Coeficiente } & \multirow{2}{*}{} & \multicolumn{2}{|c|}{$\mathrm{T}\left({ }^{\circ} \mathrm{C}\right)$} & \multicolumn{2}{c|}{$\begin{array}{c}\mathrm{Fe}_{3} \mathrm{Si} \\
\text { (no estequiométrico) }\end{array}$} & \multicolumn{2}{|}{$\mathrm{Fe}_{3} \mathrm{Si}$} \\
\cline { 3 - 7 } & & Fe(a) & Fe 5.8\%at.Si & Fe 18\%at.Si & Fe 21\%at Si & Fe 25\%at.Si \\
\hline \multirow{2}{*}{$D_{S i}\left(\mathrm{~m}^{2} / \mathrm{s}\right)$} & 800 & $1.310^{-16}$ & $2.510^{-15}$ & $1.410^{-16}$ & $3.610^{-17}$ & $1.310^{-16}$ \\
\cline { 2 - 7 } & 1125 & - & $7.710^{-13}$ & $6.410^{-13}$ & $3.010^{-13}$ & $3.510^{-13}$ \\
\hline \multirow{2}{*}{$D_{F e}\left(\mathrm{~m}^{2} / \mathrm{s}\right)$} & 800 & $410^{-16}$ & $810^{-16}$ & $6.1710^{-14}$ & $6.0810^{-13}$ & $1.5610^{-12}$ \\
\cline { 2 - 7 } & 1125 & - & $410^{-13}$ & $3.2010^{-12}$ & - & - \\
\hline
\end{tabular}

por su interés y apoyo durante el trabajo. Asimismo agradecemos al Prof. J. Degauque (INSA, Toulouse) las numerosas conversaciones mantenidas en relación con este tema.

\section{BIBLIOGRAFÍA.}

1. A.J. Moses, Electrical Steels: Past present and future developments, IEEE Proc. A137, 233-245 (1990)

F.E. Werner, CMP Report, No. 91-11, EPRI Project Number RP3070-24 (1991)

3. Y. Tanaka, A. Hiura, H. Ninomiya, K.Tsuru, H. Kobayashi, S. Masuda, Application of $6.5 \%$ Si steel sheet to audio frecuency transformers, NKK Technical Review 60, 9-15 (1990)

4. Y. Takada, M. Abe, S. Masuda, J. Inagaki, Commercial scale production of Fe-6.5wt.\%Si sheet and its magnetic properties, J. Appl. Phys. 64, 5367-5369 (1988)

5. K. Nakaoka, Y. Takada, Y. Tanaka, Patent no. US4832762, 3 de marzo de 1986

6. O. Kubachewski, Iron Binary Phase Diagrams, Springer-Velag, 136-139 (1982)

7. V. Zhang and D.G. Ivey, $\mathrm{Fe}_{3} \mathrm{Si}$ formation in Fe-Si couples, J. Mater. Sci., 33, 3131-3135 (1998)

8. A. Gude and H. Mehrer, Diffusion in $\mathrm{DO}_{3}$-type intermetallic phase $\mathrm{Fe}_{3} \mathrm{Si}$, Phil. Mag. 76A(1), 1-29 (1997)

9. J. Borj and D.Y.F. Lay, Diffusion in a-Fe-Si alloys, J. Appl. Phys., 41(13), 51935200 (1970)

10. A. Schulze, Thermal expansion of alloys of iron, Z. Tech. Physik. 9, 338-343 (1928)

11. K.E. Peterson, Silicon as mechanical material, Proc. IEEE, 70, 420 (1982). 